\title{
Estudios universitarios vs. Formación profesional en Comunicación: ¿una mejor integración laboral?
}

\section{University Degree vs. Occupational Traning: ¿a better job placement?}

Raquel Vinader Segura. Grupo FONTA - Universidad Complutense de Madrid

Natalia Abuín Vences. Grupo FONTA - Universidad Complutense de Madrid

Alberto García García. Grupo FONTA - Universidad Complutense de Madrid

Resumen:

La Universidad se enfrenta actualmente a la integración en el Espacio Europeo de Educación Superior (EEES), que busca armonizar las titulaciones superiores en la UE y, al mismo tiempo, integrar la formación práctica en todas las materias con el objetivo de preparar al alumno para el ejercicio profesional.

El objetivo de esta investigación es ofrecer un análisis de la oferta formativa en el área de la Comunicación tanto en el ámbito universitario como de formación profesional, y realizar un estudio comparativo de la inserción laboral en ambos niveles de enseñanza para determinar si la especialización y la formación práctica tienen correlación en la integración laboral de nuestros estudiantes.

Palabras clave:

Formación profesional; Universidad; inserción laboral; Estudios de Comunicación.

Abstract:

The spanish university system is currently facing the implementation of European Higher Education Area (EHEA) which main objective is to harmonize this level of education in the EU and, at the same time, to integrate the practical training in the study programmes in order to train the student for the professional practice.

This paper presents the analysis of the academic offer in the Communication area in the higher education: universities' degrees and occupational training plans; as well as a comparative study of the job placement for graduates from both type of education. The main goal is to determinate if specialization and practical training has a positive correlation for a better labour integration.

Keywords:

University degrees; Occupational Training; Job Placement; Communication studies. 


\section{Introducción}

Los estudios universitarios se enfrentan actualmente al reto de la integración en el Espacio Europeo de Educación Superior (EEES). Una serie de cambios orientados a armonizar las titulaciones superiores en todos los países de la UE y que busca, al mismo tiempo, integrar la formación práctica en todas las materias con el objetivo de preparar al alumno para el ejercicio profesional. Precisamente, una de las asignaturas pendientes de los estudios de Comunicación es la inserción laboral de sus titulados. Si atendemos los datos de contrataciones en esta área, la situación que se presenta no es muy alentadora.

Por el contrario, la Formación Profesional se presenta como una alternativa para el alumnado al ser un tipo estudios orientados al empleo. Este planteamiento teórico-práctico culmina en la realización obligatoria de prácticas laborales que ofrecen al alumnado un primer contacto con un entorno real de trabajo constituyéndose de este modo un puente entre formación y empleo.

\section{Objetivos}

El grupo de investigación FONTA (Formación en Nuevas Tecnologías del Audiovisual) de la Universidad Complutense de Madrid tiene como propósito fundamental la construcción de un puente entre la formación en Comunicación Audiovisual y el sector profesional. Parte de sus líneas de investigación pretenden propiciar un debate amplio sobre la formación, que la sociedad y la industria reclaman de la universidad.

En concreto, esta investigación tiene los siguientes objetivos:

- $\quad$ Trazar un mapa de la oferta formativa en el área de la Comunicación en nuestro país.

- Analizar la inserción laboral de los titulados en Comunicación: formación profesional y estudios universitarios.

- Realizar un estudio comparativo sobre la empleabilidad en estos dos niveles de formación en España para el área de la Comunicación.

Se trata en definitiva de dilucidar si la formación práctica de los estudiantes de Formación Profesional tanto dentro como fuera del centro educativo (Formación en Centros de Trabajo) mejora la inserción laboral de los alumnos, ofreciendo mayores posibilidades de trabajar en aquellas ocupaciones para las cuales fueron formados. 


\section{Metodología}

Establecidos los objetivos concretos de la presente investigación, hemos realizado un análisis documental que nos permite definir los diferentes niveles de formación en el área de Comunicación, así como los cambios que se están produciendo los mismos, fundamentalmente fruto de la convergencia con las políticas de la Unión Europea.

Pero, para poder realizar un análisis comparativo de la inserción laboral de los titulados en estos estudios, hemos acudido a los datos oficiales que publica el Servicio Público de Empleo Estatal (SEPE) -anteriormente Instituto Nacional de Empleo (INEM), organismo público dependiente del Ministerio de Trabajo e Inmigración "al que se le encomienda la ordenación, desarrollo y seguimiento de los programas y medidas de la Política de Empleo" (SEPE, 2011). Dentro del Servicio de Empleo, destaca la labor del Observatorio de las Ocupaciones que se encarga del análisis de la situación y las tendencias del mercado de trabajo, ofreciendo los datos actualizados sobre la inserción laboral de nuestros titulados.

Esta investigación presenta las conclusiones de un estudio comparativo que tiene en cuenta las ocupaciones en las que se contrataron en 2010 los titulados en alguno de los estudios objeto de nuestro análisis. Esto es:

- $\quad$ Familia profesional de Comunicación, Imagen y Sonido:

- Técnico de Grado Superior en Imagen

- Técnico de Grado Superior en Producción de Audiovisuales y Espectáculos

- Técnico de Grado Superior en Realización de Audiovisuales y Espectáculos

- Técnico de Grado Superior en Sonido

- $\quad$ Licenciaturas en el área de Ciencias de la Información o la Comunicación:

- Licenciado en Comunicación Audiovisual

- $\quad$ Licenciado en Periodismo

- $\quad$ Licenciado en Publicidad y Relaciones Públicas

No obstante, y ante la amplitud del objeto de estudio, con más de 250 ocupaciones distintas por especialidad, decidimos acotar la muestra de análisis a las 10 categorías laborales más contratadas por cada título. Datos que organizamos siguiendo tres criterios fundamentales: ocupaciones en el sector de la formación cursada, otras ocupaciones en la industria de la Comunicación y ocupaciones en otros sectores. 


\section{Los estudios superiores de Comunicación en España}

Los estudios universitarios en el área de la Comunicación se remontan a 1970 cuando la Ley General de Educación transforma los estudios de Periodismo y demás medios de comunicación social-hasta el momento impartidos por las escuelas profesionales de Periodismo, Cine, Radiodifusión y Publicidad-, para incorporarlos a las enseñanzas universitarias en sus tres ciclos y titulaciones: Diplomado, Licenciado y Doctor. Así, en 1971 se crean las primeras facultades en España de Ciencias de la Información, vertebradas en tres grandes ramas: Periodismo, Ciencias de la Imagen Visual y Auditiva, y Publicidad y Relaciones Públicas. Todas ellas con un primer ciclo común y un segundo ciclo diferenciado para cada tipo de estudios.

Pero, más allá de los diferentes planes de estudios aprobados desde los años 70 hasta hoy y que han ido transformando las disciplinas que se imparten en las aulas universitarias, cabe destacar el propio concepto de los estudios universitarios y que los distancian significativamente de los de Formación Profesional. Según la Ley Orgánica de Reforma Universitaria (1983), “un número creciente de estudiantes [que] exigen un lugar en las aulas, bien para su formación profesional, bien para satisfacer un creciente y loable interés por la cultura en sus diversas formas". Y es precisamente esta última concepción la que predomina en los estudios universitarios, que adolecen de materias y conocimientos prácticos necesarios para el ejercicio profesional.

Por su parte, la Formación Profesional persigue un fin concreto que rige la organización de los propios estudios, así como la manera de impartir las disciplinas que componen cada especialidad: la formación para la inserción en el mercado laboral. De este modo, los estudios de FP "capacitan para el desempeño cualificado de las diversas profesiones, el acceso al empleo y la participación activa en la vida social, cultural y económica”.

En este sentido, son significativas las modificaciones introducidas por la Ley de Ordenación General del Sistema Educativo (LOGSE) en 1990 que busca, por un lado, facilitar la integración otros niveles de enseñanza (secundaria, formación profesional y universidad) abriendo nuevas vías de acceso para los estudiantes. La organización modular de los ciclos formativos (grado medio y superior) permite la flexibilización de los itinerarios formativos, posibilitando el acceso a los estudios universitarios.

Por otro lado, busca la conexión de los titulados en estos estudios con el mercado productivo mediante la "Formación en Centros de Trabajo" (FCT) que "consiste en la realización de actividades productivas propias del perfil profesional del título a conseguir" (Consejo Superior de Cámaras de Comercio, 2001), Es decir, un módulo práctico que se cursa fuera de las instalaciones del centro educativo, en un entorno real de trabajo (una empresa del sector), donde el alumno tiene la posibilidad de aplicar lo aprendido y evidencia las competencias adquiridas. Una posibilidad contemplada en planes de estudio previos. La diferencia radica en que la LOGSE hace obligatorio que, cualquier alumno que desee obtener su título, deba cumplir obligatoriamente con este período de prácticas formativas en centros de trabajo. 
Por tanto, la Formación Profesional tiene por finalidad preparar a los alumnos para la actividad en un campo profesional y facilitar su adaptación a las modificaciones laborales que puedan producirse a lo largo de su vida.

El Real Decreto 1128/2003, que regula el Catálogo Nacional de Cualificaciones Profesionales, estructura la Formación Profesional en España en 26 familias profesionales atendiendo a criterios de afinidad de la competencia profesional. La familia de Comunicación, Imagen y Sonido ofrece las especialidades de Imagen, Producción, Realización y Sonido, cubriendo, de este modo, las principales ocupaciones del sector audiovisual. Mientras que, por el contrario, no se recogen titulaciones que se equiparen con los estudios de Periodismo o Publicidad.

A su vez, la Formación Profesional se encuentra inmersa en una serie de transformaciones encaminadas a adaptar los títulos y especialidades a las nuevas necesidades del mercado y ofreciendo un sistema de estudios más flexible que permite la formación presencial, a distancia o mixta, poniendo al alcance de todos la posibilidad de actualizar su formación o conseguir una titulación oficial. Así, en noviembre de 2011 se aprobaron los títulos de Técnicos Superiores en Iluminación, Captación y Tratamiento de la Imagen y en Animaciones 3D, Juegos y Entornos Interactivos, que pasan a incorporarse a esta Familia Profesional, mientras que el Técnico Superior en Marketing y Publicidad se integrará en la Familia de Comercio y Marketing.

Cuadro I. La Formación Profesional en el sistema educativo

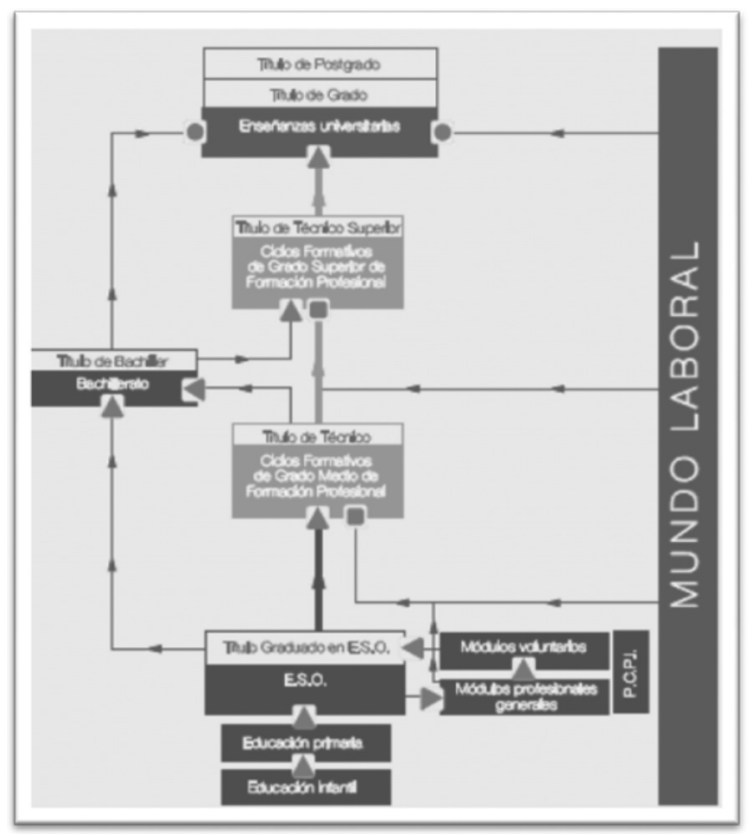

Fuente: Ministerio de Educación, Cultura y Deporte (2001). 


\section{La empleabilidad en los estudios de Comunicación (2009): estudio comparativo}

A continuación, procedemos a analizar los datos de inserción laboral para los estudios de Educación Superior relacionados con el sector de la Comunicación:

\subsection{Estudios universitarios}

Durante 2009 y según datos del Observatorio Ocupacional del Servicio Público de Empleo (SEPE) se realizaron 17.201 contratos, de los cuales 7.886 correspondieron a licenciados en Periodismo, 5.158 a Comunicación Audiovisual y 4.157 a Publicidad y Relaciones Públicas, como muestra el siguiente gráfico.

Se consolida así una tendencia de empleabilidad entre este tipo de titulados creciente desde 2008, en que se recogieron los niveles más bajos de empleo de los últimos años, y superando de este modo los datos ofrecidos en 2005.

Cuadro II. Número de contratos en Licenciados en Comunicación según especialidad (2009).

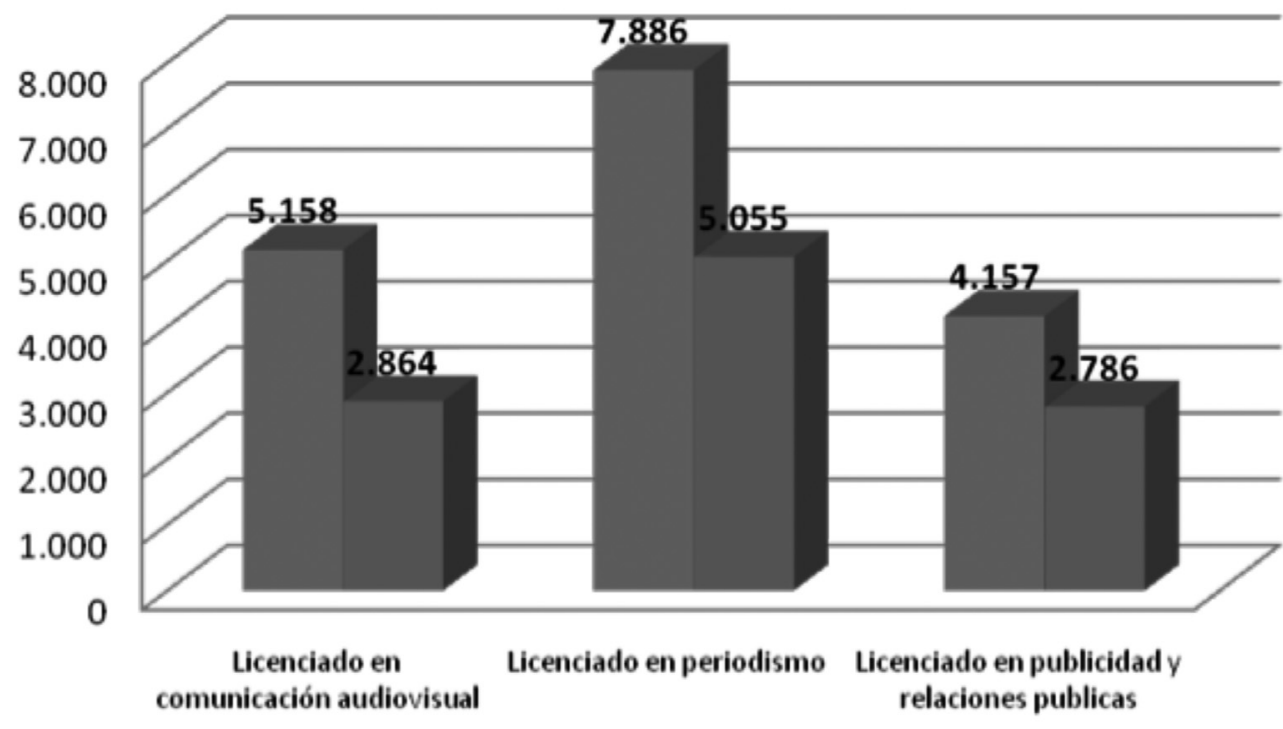

Contratos Personas Contratadas

Fuente: Elaboración propia sobre datos de INEM 
No obstante, estos datos, lejos de ser positivos, pueden ser explicados por el alto grado de inestabilidad y movilidad laboral de este tipo de trabajadores. De esta manera, si atendemos al ratio contratos-personas contratadas durante 2010, observamos se realizaron de media hasta 1.8 contratos (Comunicación), 1.56 (Periodismo) y 1.50 (Publicidad). Estas cifras vienen refrendadas por el alto nivel de temporalidad de los contratos que se realizaron en este año. Así, únicamente menos de un $16 \%$ del total de los contratos que se realizaron en el área de Publicidad tenían carácter de indefinido, una cifra significativamente más baja en el área de Comunicación. Entre este tipo de licenciados, el 93.7\% de los contratos celebrados en 2009 tuvieron una duración temporal.

En cuanto a la distribución geográfica de las contrataciones, Madrid se consolida claramente como la ciudad española donde se realizan la mayor parte de las contrataciones de los licenciados en el área de Comunicación, fundamentalmente se debe a que en la Capital están establecidas las sedes centrales de los principales Grupos de Comunicación. De este modo, el 47,6\% de los licenciados en Comunicación se contrata en la Comunidad de Madrid, seguido de Periodismo $(38,9 \%)$ y Publicidad y Relaciones Públicas $(35,45 \%)$. Siguen, por volumen de contratos, ciudades como Valencia, Vizcaya o Barcelona.

Cuadro III. Distribución geográfica de la contratación de los Licenciados en Comunicación (2009).

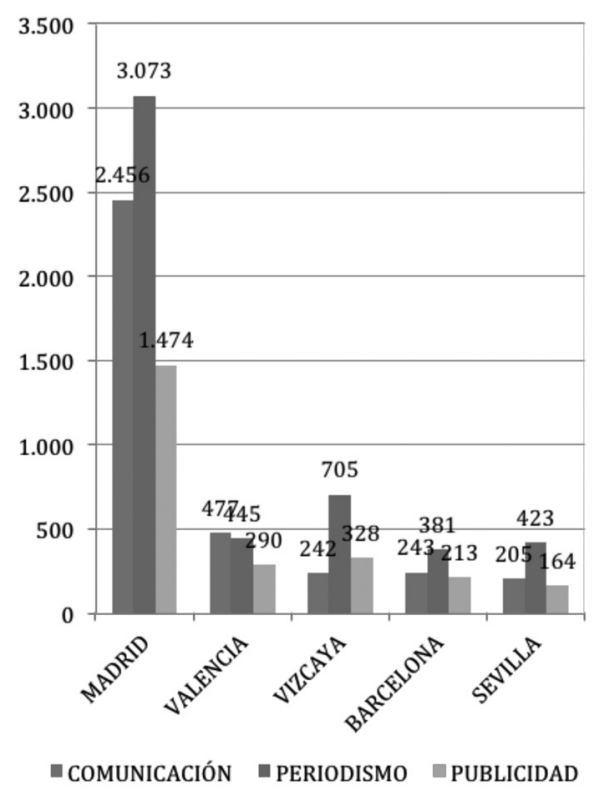

Fuente: elaboración propia sobre datos INEM 
En cuanto al perfil del contratado, los datos ofrecidos por el Servicio Público de Empleo (SEPE) muestran una mayor contratación de mujeres de todas las especialidades pero es más significativo entre los titulados en Publicidad y Relaciones Públicas, donde tres cuartas partes de las contrataciones corresponden al género femenino. Esto puede deberse al mayor peso de las mujeres tanto en alumnos matriculados como titulados.

En cuanto a la edad, los contratos corresponden de manera mayoritaria para los segmentos de edad comprendidos entre los 25-29 y 30-39 años. Por especialidades, únicamente resaltar que los perfiles más jóvenes tienen más peso en las titulaciones de Comunicación y Publicidad frente a Periodismo donde predomina la contratación de titulados de 30 a 39 años de edad.

Sobre las ocupaciones contratadas debemos destacar, como se muestra a continuación, el 52,42\% de los titulados en Comunicación Audiovisual consiguieron un contrato dentro del sector audiovisual; un 14,56\% lo hicieron en el área de Periodismo, mientras que un 20,83\% lo hicieron en otras ocupaciones ajenas al sector como, por ejemplo, 'vendedores en tiendas y almacenes', 'camareros', grabadores de datos' o 'azafatos de tierra'. Un porcentaje que podría verse aumentado si tenemos en cuenta que el 8\% de los contratos de los egresados en Comunicación Audiovisual, recogidos bajo epígrafes de administrativos, no pueden computarse en ninguna de las categorías anteriores.

Por su parte, las ocupaciones relacionadas con el área de estudio de Periodismo supusieron en 2009 el 40,9\% de las contrataciones, incrementándose con el 8,93\% de los periodistas que consiguieron emplearse como 'Actores'. El resto de las ocupaciones más contratadas se completan con un 15,96\% como administrativos y un $34,20 \%$ en otras categorías como 'Telefonistas' o 'Azafatos de tierra'. 
Tabla I. Ocupaciones más contratadas entre los licenciados en Comunicación.

\begin{tabular}{|c|c|c|c|c|}
\hline & & COMUNICACIÓN & PERIODISMO & PUBLICIDAD \\
\hline 1 & Actores & 777 & 447 & 77 \\
\hline 2 & Agentes y representantes comerciales & 93 & 0 & 291 \\
\hline 3 & Asistentes de dirección y adminis tra tivos & 166 & 278 & 231 \\
\hline 4 & Azafatos de tierra & 160 & 232 & 216 \\
\hline 5 & Camareros asalariados & 189 & 0 & 206 \\
\hline 6 & $\begin{array}{l}\text { Empleados administrativos sin tareas de atención al } \\
\text { público no clasificados bajo otros epigrafes }\end{array}$ & 255 & 576 & 420 \\
\hline 7 & Grabadores de datos & 170 & 321 & 244 \\
\hline 8 & Locutores de radio, TV y otros presentadores & 91 & 221 & 0 \\
\hline 9 & Periodistas & 515 & 2.188 & 167 \\
\hline 10 & Profesionales de la publicidad y la comercialización & 49 & 0 & 219 \\
\hline 11 & Recepcionistas (excepto de hoteles) & 82 & 0 & 165 \\
\hline 12 & Técnicos de grabación audiovisual & 812 & 229 & 0 \\
\hline 13 & Técnicos de radiodifusión & 397 & 0 & 0 \\
\hline 14 & Telefonistas & 121 & 245 & 0 \\
\hline 15 & Vendedores en tiendas y almacenes & 379 & 528 & 487 \\
\hline
\end{tabular}

Fuente: Elaboración propia sobre datos de INEM.

Una situación algo más compleja es la que presentan los titulados en Publicidad y Relaciones Públicas, quienes sólo consiguieron colocarse en un $17,7 \%$ en ocupaciones relacionadas con sus estudios superiores. Al mismo tiempo, nos encontramos con contratos como administrativos (16,10\%) pero son el resto de ocupaciones ('Vendedores', 'Representantes comerciales', 'Azafatos’ o ‘Grabadores de datos') los que consiguen aglutinar el grueso de la contratación (66,15\%).

\subsection{Formación Profesional}

Este tipo de titulados consiguieron generar, según los datos del Observatorio de las Ocupaciones (SEPE, 2009), 10.998 contratos de los cuales $40 \%$ correspondió a la especialidad de Imagen, 35\% para Realización, 13\% Sonido y, por último, 11\% para producción. Sin embargo, al contrastar estos datos con el número de personas contratadas, nos encontramos que, por término medio, cada titulado en Formación Profesional, tuvo 2,3 contratos en ese año. 
Cuadro IV. Distribución del empleo entre los Técnicos de Formación Profesional.

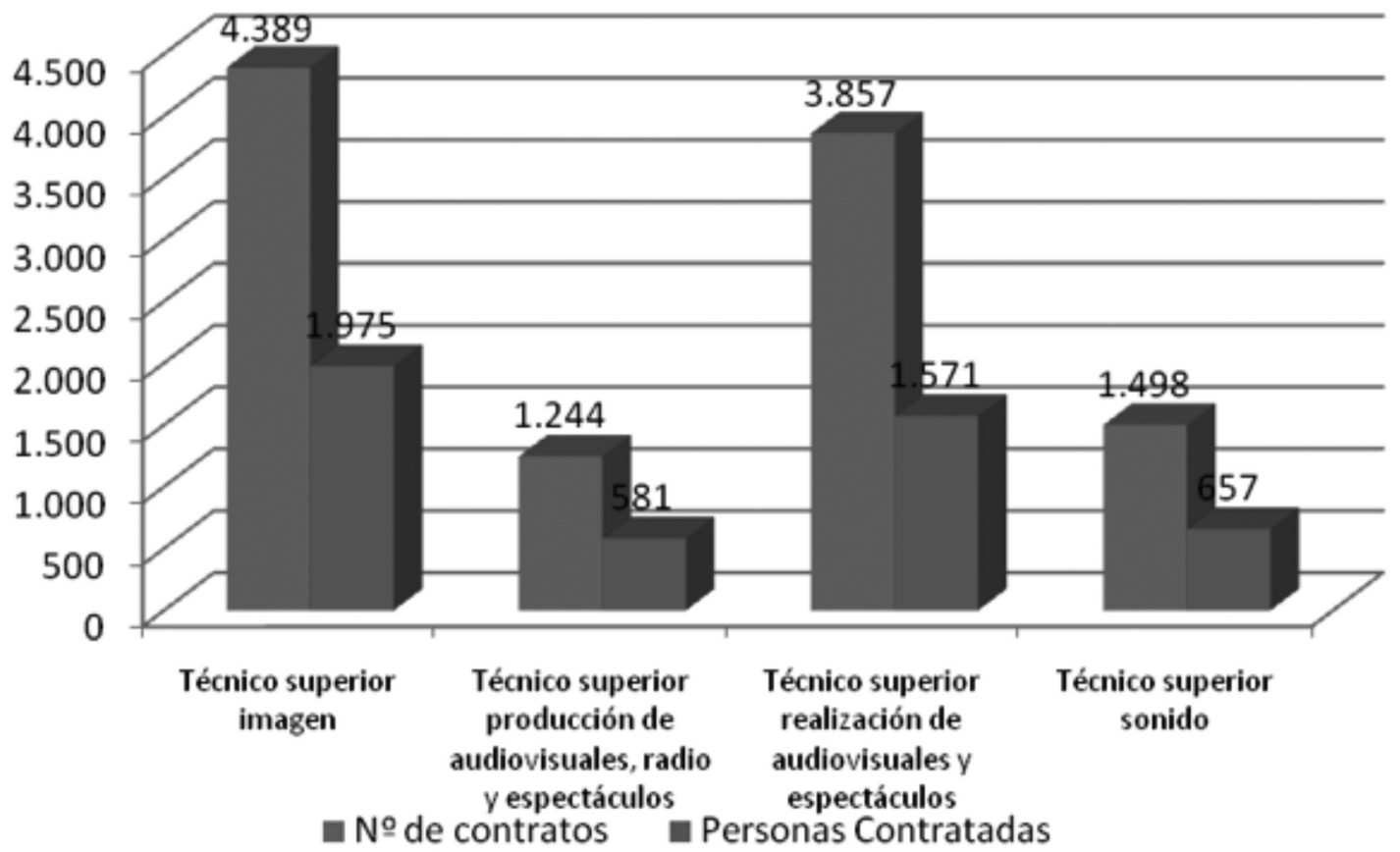

Fuente: elaboración propia sobre datos de INEM.

Estos mismos datos nos proporcionan un perfil algo distinto del de los licenciados que se contrataron en el período estudiado. De este modo, de las 10.988 personas contratadas en 2009 el 63\% eran varones con un rango de edad que oscilaba entre los 30 y 39 años de edad.

En cuanto a las ocupaciones en las que se contrataron hay que destacar que el alto nivel de ocupación en el sector audiovisual con más del $66 \%$ en el caso de los técnicos en producción hasta el $95 \%$ de los técnicos en Imagen. Además, si analizamos las 10 contrataciones más demandadas por parte de las empresas, nos encontramos que existe movilidad entre las diferentes especialidades. Así, como muestra el gráfico, excepto ocupaciones específicas como, por ejemplo, "Dirección de departamento de producción" o "montadores de equipos electrónicos", otras categorías laborales son desempeñadas indistintamente por los técnicos superiores. Nos referimos a "Fotógrafos y operadores de equipos de grabación", "operadores de equipos de radio y televisión" o "actores y directores de cine, radio y televisión". 
Tabla II. Ocupaciones más contratadas en Técnicos Superiores en Comunicación según especialidad (2009).

\begin{tabular}{|c|c|c|c|c|c|}
\hline & & IMAGEN & PROD & REALIZ & SONIDO \\
\hline 1 & FOTÓGRAFOS Y OPERADORES DE EQUIPOS DE GR & 749 & 146 & 710 & 320 \\
\hline 2 & OPERADORES DE EQUIPOS DE RADIO Y TELEVIS & 543 & 96 & 607 & 92 \\
\hline 3 & DEPENDIENTES Y EXHIBIDORES EN TIENDAS, A & 273 & 80 & 201 & 67 \\
\hline 4 & ACTORES Y DIRECTORES DE CINE, RADIO, TEL & 226 & 101 & 252 & 0 \\
\hline 5 & PEONES DE INDUSTRIAS MANUFACTURERAS & 162 & 89 & 89 & 135 \\
\hline 6 & CAMAREROS, BÁRMANES Y ASIMILADOS & 159 & 58 & 91 & 43 \\
\hline 7 & PEONES DEL TRANSPORTE Y DESCARGADORES & 147 & 44 & 103 & 70 \\
\hline 8 & ANIMADORES COMUNITARIOS & 89 & 0 & 0 & 0 \\
\hline 9 & TAQUÍGRAFOS Y MECANÓGRAFOS & 88 & 0 & 0 & 0 \\
\hline 10 & AUXILIARES ADMINISTRATIVOS CON TAREAS DE & 80 & 30 & 0 & 0 \\
\hline 11 & DIRECCIÓN DE DEPARTAMENTO DE PRODUCCIÓN & 0 & 49 & 0 & 0 \\
\hline 12 & SECRETARIOS ADMINISTRATIVOS Y ASIMILADOS & 0 & 34 & 0 & 0 \\
\hline 13 & TÉCNICOS EN ELECTRÓNICA Y TELECOMUNICACI & 0 & 0 & 128 & 42 \\
\hline 14 & TÉCNICOS EN ELECTRICIDAD & 0 & 0 & 72 & 37 \\
\hline 15 & TELEFONISTAS & 0 & 0 & 67 & 0 \\
\hline 16 & MONTADORES DE EQUIPOS ELECTRÓNICOS & 0 & 0 & 0 & 56 \\
\hline 17 & AJUSTADORES Y REPARADORES DE EQUIPOS ELE & 0 & 0 & 0 & 47 \\
\hline
\end{tabular}

Fuente: elaboración propia sobre datos de INEM. 
Tabla III. Cuadro comparativo de las 20 ocupaciones más contratadas para Licenciados y Técnicos Superiores en Comunicación (2009).

\begin{tabular}{|c|c|c|c|c|c|c|c|c|c|}
\hline & & COMUNICACIÓN & PERIODISMO & PUBLICIDAD & IMAGEN & PRODUCCIÓN & REALIZACIÓN & SONIDO & Total \\
\hline 1 & Periodistas & 515 & 2.188 & 167 & 36 & 9 & 53 & 5 & 2.973 \\
\hline 2 & Vendedores en tiendas y almacenes & 379 & 528 & 487 & 273 & 80 & 201 & 67 & 2.015 \\
\hline 3 & Fotografos y operadores de equipos de gr & & & & 749 & 146 & 710 & 320 & 1.925 \\
\hline 4 & Actores & 777 & 447 & 77 & 226 & 101 & 252 & 0 & 1.880 \\
\hline 5 & $\begin{array}{l}\text { Empleados administrativos sin tareas de atención al } \\
\text { público no clasificados bajo otros epigrafes }\end{array}$ & 255 & 576 & 420 & 39 & 25 & 36 & 8 & 1.359 \\
\hline 6 & Operadores de equipos de radio y televis & & & & 543 & 96 & 607 & 92 & 1.338 \\
\hline 7 & Técnicos de grabación aucliovisual & 812 & 229 & 0 & & & & & 1.041 \\
\hline 8 & Grabadores de datos & 170 & 321 & 244 & 14 & 3 & 6 & 4 & 762 \\
\hline 9 & Camareros as alariados & 189 & 0 & 206 & 159 & 58 & 91 & 43 & 746 \\
\hline 10 & Peones de industrias manufactureras & 79 & 89 & 90 & 162 & 89 & 89 & 135 & 733 \\
\hline 11 & Azafatos de tierra & 160 & 232 & 216 & 41 & 10 & 62 & 10 & 731 \\
\hline 12 & Asistentes de dirección y administrativos & 166 & 278 & 231 & & & & & 675 \\
\hline 13 & Peones del transporte y descargadores & 50 & 58 & 35 & 147 & 44 & 103 & 70 & 507 \\
\hline 14 & Telefonistas & 121 & 245 & 0 & 0 & 0 & 67 & 0 & 433 \\
\hline 15 & Técnicos de radilodifusión & 397 & 0 & 0 & & & & & 397 \\
\hline 16 & Agentes y representantes comerciales & 93 & 0 & 291 & & & & & 384 \\
\hline 17 & Locutores de radio, TV y otros presentadores & 91 & 221 & 0 & 26 & 4 & 18 & 4 & 364 \\
\hline 18 & Recepcionistas (excepto de hoteles) & 82 & 0 & 165 & 28 & 5 & 24 & 4 & 308 \\
\hline 19 & \begin{tabular}{|l} 
Profesionales de la publicidad y la \\
comercialización \\
\end{tabular} & 49 & 0 & 219 & & & & & 268 \\
\hline 20 & Técnicos en electrónica y telecomunicaci & 18 & 13 & 6 & 0 & 0 & 128 & 42 & 207 \\
\hline
\end{tabular}

Fuente: elaboración propia sobre datos de INEM.

\section{Resultados}

Atendido a las cifras expuestas en el apartado anterior, debemos resaltar los siguientes aspectos:

- $\quad$ Además de las diferencias en los tipos de estudios, encontramos un perfil específico para cada tipo de enseñanza. El perfil del Licenciado en Comunicación es una mujer de 25 a 29 años con estudios de Periodismo o Comunicación, mientras que el Técnico de Grado Superior es un hombre de 30 a 39 años especializado en Imagen o Realización. 
Los estudios universitarios supusieron en 2009 más personas contratadas que los de Formación Profesional. De este modo, 10.705 personas se emplearon en este año frente a 4.784 que acreditaron tener estudios de FP. Sin embargo, si observamos el número de contrataciones, 17.2501 para licenciados frente a 10.838 de los Técnicos, nos encontramos con una circunstancia interesante. Los titulados en Formación Profesional tuvieron más contratos por término medio que los licenciados, lo que implica que existe mayor movilidad laboral entre este tipo de profesionales. Cada técnico superior tuvo de media 2,27 contratos durante el período estudiado; una cifra que se reduce en el caso de los licenciados (1.62).

- Un aspecto relacionado es la temporalidad de la contratación en los egresados en Comunicación, una situación que se presenta de la misma manera en ambos tipos de titulados en esta área de conocimiento. Así, en 2009 se realizaron 7.758 contratos de media con una duración temporal y 775 con carácter indefinido. No obstante, debemos resaltar que el 95,8\% de los contratos de los titulados en Formación Profesional tuvo una duración determinada. Este hecho es probablemente debido a las propias características del sector audiovisual y a la coyuntura económica del país.

- En cuanto a las ocupaciones en las que se contratan los titulados en Comunicación también podemos apreciar diferencias significativas en función del tipo de estudios cursados. De este modo, debemos resaltar que el 81,05\% de los titulados en cualquiera de las especialidades de la Familia Profesional de Comunicación, Imagen y Sonido consiguió en 2009 un empleo en el sector audiovisual, área de actividad natural para sus estudios, mientras que en el caso de los estudiantes universitarios este nivel de contratación únicamente ascendió apenas al 45\%.

- La contratación fuera del sector audiovisual, en otras actividades no relacionadas con la Comunicación, supuso el 40,39\% de los contratos entre los licenciados, cifra que se reduce hasta el 18\% en el caso de los técnicos superiores.

- Teniendo en cuenta las especialidades de estudios, las cifras de empleabilidad en el sector de la Comunicación ascienden todavía más en el caso de los titulados de FP en Imagen (94,7\%) y Realización (90,23\%). En el caso de los estudios universitarios, Comunicación Audiovisual es la carrera con mejor inserción con mejor inserción en su sector con un $67 \%$.

- Las peores cifras corresponden a los egresados en Publicidad y Relaciones Públicas con un 17,74\% de ocupaciones en el sector audiovisual. Por parte de la Formación Profesional, el Técnico Superior con Producción arroja las cifras más bajas con un 66,85\% de contratos en su área de actividad, prácticamente lo mismo que los titulados universitarios con mayor índice de contratación en el área de Comunicación.

Ante todas estas evidencias, y retomando los objetivos de la presente investigación, podemos concluir que el área actividad en la industria de la Comunicación se halla cubierta por estudios superiores, tanto en el ámbito universitario como 
en el de la Formación Profesional. Si bien el mercado final es el mismo, los objetivos y medios de los que dispone cada tipo de enseñanza difieren significativamente, lo que necesariamente debe tener su correlato en las cifras finales de empleabilidad.

Una formación especializada, adaptada a las herramientas tecnológicas que requiere el mercado, así como la obligatoriedad de cursar al menos una estancia formativa en una empresa del sector colocan a los Técnicos de Grado Superior en una situación de ventaja a la hora de encontrar un empleo acorde a las expectativas de estos titulados.

Aunque la inserción laboral de los licenciados difiere en función de la especialidad, las bajas tasas de empleabilidad en el sector audiovisual deben abrir un debate crítico sobre hacia dónde deben dirigirse nuestros estudios universitarios de Comunicación. La meta laboral en el sector de referencia no debe ser considerada por algunos como rendirse a la economía y a sus agentes. La carrera docente y académica debe ser una opción y no el último recurso ante el empleo en sectores externos a nuestra área de conocimiento.

\section{Referencias bibliográficas}

Dirección General de Universidades (2010): Datos sobre facultades de Comunicación y alumnos matriculados/titulados. Ley Orgánica, 11/1983, de 25 de agosto, de Reforma Universitaria (LRU). Publicada en el B.O.E. de 1 de septiembre de 1983. Ministerio de Educación, Cultura y Deporte (2001): Manual de Formación en Centros de Trabajo. Madrid: Consejo Superior de Cámaras de Comercio.

Observatorio de las Ocupaciones (SEPE) (2010): Datos sobre el mercado de trabajo de los titulados en las disciplinas de Ciencias de la Información y la Familia Profesional de Comunicación, Imagen y Sonido.

Vivar Zurita, H. (Coord.) (2010): Los estudios de comunicación en España. Reflexiones en torno al Libro Blanco. Madrid: Icono 14. 